\title{
Integration of genetics into clinical teaching in medical school education
}

Bruce R. Korf, $M D, P h D$

\begin{abstract}
Medical genetics has moved from the study of rare conditions to the illumination of disorders that impact the entire spectrum of medical practice. While there have been a number of predictions and concerns about this impact, this article examines three areas where medical genetics is clearly an important tool in medical practice. First, a family history aids in risk assessment, even in common disorders that are multifactorial. Second, by elucidating molecular pathways, gene identification may lead to the development of more efficacious medications that have fewer side effects. Third, an awareness of population-based risk and the availability of genetic screening in these populations will help physicians assess an individual patient's risk. To fully benefit from genetically based medical approaches, physicians will need to master a new set of principles and clinical skills. However, genetics has traditionally been taught as a basic science, sometimes under the purview of cell biology or biochemistry. Often, then, genetics has little or no place in clinical teaching. This article describes an effort at Harvard Medical School to integrate genetics into both the preclinical and the clinical curricula. The author looks at the underlying pedagogy, how basic science teaching in genetics is provided, and an approach currently being used to include genetics in clinical teaching.
\end{abstract}

Genet Med 2002:4(6, Supplement):33S-38S.

Key Words: medical genetics, education, medical school, teaching

Medical genetics has turned a corner and has moved from the study of rare conditions to the illumination of disorders that impact on the entire spectrum of medical practice. Genetics will increasingly occupy a central role in the practice of medicine, requiring physicians to master a new set of basic principles and clinical skills. This will necessitate a shift in emphasis in medical education to prepare the current generation of practitioners. Currently, genetics is commonly taught as a basic science, sometimes in a free-standing course, but often as part of a larger course, such as biochemistry or cell biology. There is typically little attention directed toward genetics in clinical teaching, leaving the student to conclude that genetics is largely irrelevant to clinical practice. In this article I will describe an effort at Harvard Medical School to integrate genetics into both the preclinical and clinical curricula. We will look at the underlying pedagogy, how basic science teaching in genetics is provided, and an approach being used to include genetics in clinical teaching.

\section{Genetics in medical practice}

How will genetics impact the practice of medicine? The traditional focus of medical genetics is on the diagnosis and man-

\footnotetext{
From the Harvard-Partners Center for Genetics and Genomics, Boston, Massachusetts.

Bruce R. Korf, MD, PhD, Harvard-Partners Center for Genetics and Genomics, 77 Avenue

Louis Pasteur, Boston, MA 02115.

Received: July 30, 2002

Accepted: September 23, 2002.
}

DOI: 10.1097/01.GIM.0000040330.16059.98 agement of disorders due to changes in single genes or chromosomes. Major advances in individual and public health have ensued. Highlights include the elucidation of syndromes due to chromosomal imbalance, development of approaches to prenatal diagnosis, programs of newborn screening for inborn errors of metabolism, and, more recently, the advent of molecular diagnostic testing. These advances have had a major impact on the care of rare disorders, which is important for individuals and families who live with them, but have been of marginal importance in the mainstream of medicine. The teaching of genetics in medical school has been correspondingly marginalized, confined to basic science courses, often incorporated into other courses such as biochemistry.

It has long been recognized that genetic factors contribute to more common disorders, such as hypertension, diabetes, and cancer, but the etiologies of these disorders have proved to be dauntingly complex. Multiple genes contribute to risk, combined with environmental factors. Moreover, the particular genes that may be important for a given individual may be different from person to person. Until recently, tools were not at hand to decipher the genetic contribution to common disorders. This has changed, however, largely due to the efforts of the Human Genome Project. Although it will be a long time before the functions of all of the 30,000 or more genes and the 100,000 or more proteins are known, we now have at hand the ability to determine whether specific genes are involved in the pathogenesis of particular disorders. The genetics of complex disorders remains a complex problem, but it is now one that is at least tractable. 
In the excitement that has accompanied the announcement of the sequencing of the genome, some ambitious predictions have been made. ${ }^{1}$ It has been proposed that physicians will use genetic testing routinely to determine the disorders that their patients will someday develop in order to prescribe medications or recommend changes of lifestyle that prevent these conditions. Others have been concerned that genetic testing will lead to a new class of underprivileged individuals, who are subject to loss of insurance or employment based on their genetic legacy. The power of prenatal testing has been extrapolated to the selection of physical and mental traits in the unborn child, or even to a genetic reengineering of the child to instill desired traits and remove undesired ones.

Many of these predictions have been questioned, both on ethical grounds and on grounds of practicality. It has been proposed, for example, that the genetic contributions to common disorders will prove to be so complex as to impede the accuracy of predictive testing. ${ }^{2}$ Environmental factors may be of such importance that no genetic test can be predictive. Moreover, the specific combination of genetic factors in a given individual may be so unique that no two persons are alike, making it impossible to accumulate sufficient numbers of persons who share a set of genetic markers to determine outcomes on statistically meaningful samples. Gene therapy and manipulation remain elusive goals as well.

Nevertheless, there are several areas in which the impact of medical genetics on medical practice is already becoming clear. First, family history provides an important tool for risk assessment that will be increasingly useful in medical practice. Even if common disorders are usually determined multifactorially, there are some families in which mutations in individual genes are sufficient to determine a phenotype. Examples are autosomal dominant cardiomyopathy, breast and ovarian cancer due to BRCA1 or BRCA2 mutations, colon cancer due to DNA mismatch repair gene mutation, hemochromatosis, and venous thrombosis. In the case of breast cancer, $7 \%$ of affected individuals have a hereditary cause. The single gene families may be rare, but these disorders in the aggregate are common enough that most physicians will encounter them on a regular basis. Moreover, the public is becoming attuned to these conditions, and individuals may become concerned about their family history and, in some cases, overestimate their risk. It will be important, therefore, for physicians to be aware of family history and to know how to further evaluate a patient on the basis of familial risks. This will include knowing when and how to arrange genetic testing, knowing how to interpret results, understanding the significance of negative results, and being able to distinguish mutations from benign variants. They will also need to know when and how to refer patients to specialists who have more experience in medical genetics.

Second, the identification of genes that contribute to common disorders is revealing the molecular pathways that contribute to normal homeostasis and the perturbations that underlie disease. Even if a genetic contribution is too small to be useful as a genetic test, the fact that the gene makes a contribution helps to elucidate the underlying molecular process. This information, in turn, may lead to the development of new medications that more precisely target disease mechanisms with improved efficacy and reduced side effects. The result will be a new pharmacopoeia and with it the need for physicians to understand the basis for choosing a specific drug. This choice may be based on genetic testing, determining the specific etiology of a patient's disorder, and choosing the appropriate drug. Genetic testing may also be used to determine a person's pattern of drug metabolism, allowing dosage to be optimized. Also, individuals at risk of idiosyncratic adverse reactions can be identified in advance, permitting drugs that might otherwise be withdrawn from use to be used safely in a subset of patients.

Third, physicians will need to recognize the importance of population of origin in assessing genetic risk. There are a number of long-standing population screening programs, such as those for Tay-Sachs disease in Ashkenazi Jewish individuals ${ }^{3}$ or globin disorders in those of African, Mediterranean, or Asian descent. ${ }^{4,5}$ Physicians will need to be aware of populationbased risks and of the availability of genetic screening in these populations. On the other hand, studies of genetic variation within and between populations show that conventional notions of "race" have no biological meaning.

\section{Objectives: Genetics in medical education}

It will not be necessary for students to master all of the intricacies of the field to use genetics in their future practice. Genetics can be used in day-to-day practice without a detailed understanding of all of the underlying science, just as physicians can order and interpret electrolyte values without understanding the chemistry that is used in the assays. Nevertheless, there is a set of concepts and skills that will be important if the tools are to be used wisely.

The pace of change of knowledge of genetics is extraordinarily fast, yet there are some basic concepts that may be timeless and need to be understood by every physician. Some of these concepts are as follows:

1. How does a change in a gene lead to the alteration in function of a gene product, which in turn leads to an observable phenotype? The distinction between mutations in genes that encode enzymes from those that occur in structural genes needs to be recognized. An understanding of mutation implies also an understanding of the basic structure of DNA and of genes, and the processes of transcription, translation, DNA replication, and DNA repair.

2. What are the basic patterns of genetic transmission, including Mendelian genetics, mitochondrial transmission, and complex patterns due to imprinting, epistasis, etc., and how are these explained at the molecular level? Genetic mechanisms, such as gain or loss of function, dominant negative, and triplet repeat expansion need to be understood. 
3. How is the human genome organized, and what has been learned about the basis for genetic variation?

4. What is the structure and function of chromosomes, and what kinds of disorders result from abnormalities of chromosome number or structure?

5. How do multiple genes and environmental factors interact to produce multifactorial disorders, and how can we understand the genetic contributions to common disorders?

6. What is the basis for the genetic contribution to cancer, including both inherited and acquired genetic changes?

7. How do genes control the process of embryonic development, and what kinds of disorders result from perturbations in this process?

8. What are the forces that mold gene frequencies in the population, and how does this knowledge provide guidance for assessment of individual risk and establishment of population screening programs?

9. What is the ethical and legal context in which genetics is being incorporated into medical practice?

In addition to mastery of these concepts, there is a set of skills that physicians will need to acquire. These include the following:

1. The ability to obtain a family history and to recognize the major patterns of genetic transmission.

2. Awareness of the indications for chromosomal analysis and ability to interpret the reports of chromosomal abnormalities.

3. An understanding of the indications for molecular genetic testing and an ability to interpret test results.

4. Appreciation of the major approaches to prenatal diagnosis, including indications and limitations.

5. Knowledge of the role of genetics in the pathogenesis of cancer, and opportunities for genetic testing to refine estimation of risk based on family history.

6. Awareness of population screening programs, including both newborn screening for metabolic disorders and carrier screening programs.

7. Cognizance of the types of medical professionals involved in medical genetics and of the genetic counseling paradigm.

8. Sensitivity to the issues of privacy, discrimination, and family issues that arise in dealing with genetic conditions.

9. Awareness of where to go for up-to-date information on genetics, genetic testing, and genetic disorders that can be applied at the point of care.

\section{Pedagogy}

Medical schools vary widely in educational approach, and medical students are, likewise, a diverse lot. Indeed, the goals of medical education are not always the same; students may be preparing for careers in primary care, specialty practice, research, or public health, for example. Medical schools also differ in size of faculty and mix of research and clinically oriented faculty. Finally, schools differ in educational philosophy and curricular design. All of this implies that no single system of pedagogy will work at all schools, so multiple models will need to be developed and the approach used at one school may have limited ability to be translated to another.

Schools often make a distinction between basic science and clinical teaching, and many separate the two, such that the first 1 to 2 years are devoted to basic science and the latter years to clinical teaching. It is common to integrate at least some clinical teaching into the preclinical curriculum, and some have carried the integration further to the point where preclinical and clinical teaching are intermixed.

To the extent that a distinction can be made in preclinical and clinical teaching, however, there may be differences in pedagogical approaches that are relevant to the teaching of genetics. Basic science is often taught by faculty who are not clinicians and can leave students struggling to appreciate the relevance to their future clinical careers. That is not so much the case with anatomy, which is usually not taught in undergraduate courses and has obvious importance for clinical medicine, but can be a major problem with genetics. It would be easy for students to leave a genetics course believing that they have heard a rehash of undergraduate science or that genetics is a subdiscipline of biochemistry and to not appreciate the revolution in clinical medicine that is being fomented by genetics and genomics. There are a few pedagogical principles that may help to avoid this outcome.

1. Introduce major concepts with clinical cases. Students may have little understanding of the clinical approach at this stage of their education, but clinical cases can be crafted in a way that students can understand and that will illustrate why the basic concepts of genetics are important to clinical medicine.

2. Present questions before answers. The enormous growth of knowledge of basic genetic mechanisms and the esoteric but very powerful armamentarium of technologies can be intimidating. To the nongeneticist it will not be obvious why these are important unless the teaching is accompanied by a recognition of the clinical or biological question that is being addressed with a particular experimental approach. It should not be taken for granted that these questions are obvious, and students should not have to take it on faith that these approaches are important.

3. Emphasize problem-solving approaches. Most of the specific knowledge of genetics taught early in the medical school curriculum will become obsolete quickly, probably before the student graduates. Genetics is a fast-moving field and students mostly need to have the tools that will enable them to approach problems in a systematic manner and to know where to find up-to-date information when they need it.

4. Genetics is a discrete discipline. It overlaps with many other areas of basic science and medicine and therefore lends itself well to integration with other courses. There 
is, however, a body of knowledge and skills that is unique to genetics. Students should be exposed to this as a discrete course offering somewhere in the curriculum so that this knowledge does not become invisible.

In contrast with basic science teaching, genetics will almost certainly need to be integrated into other disciplines in clinical teaching. Indeed, this is desirable, in order to show how genetics contributes to the practice of medicine. Here, too, there are a number of pedagogical points that should be considered:

1. Teach by example. Students are immersed in learning to work as part of a team in the care of patients. If genetics teaching is confined solely to didactic sessions, the message is communicated that genetics does not play a critical role in patient care. Geneticists must be willing to become part of the care team, contributing to bedside teaching.

2. Use real cases for teaching. Students are becoming accustomed to using made-up cases in their preclinical courses but should expect that real cases are the basis for teaching in the clinical years.

3. Let students be the source of case material. To the maximum extent possible, use cases being seen by students on the wards or in clinics as the basis for the teaching of genetics. Use of canned cases again creates the impression that genetics is an academic subject that is not practically useful.

\section{Teaching of genetics at Harvard Medical School}

Recognizing the limitations in extrapolating a model from one school to another, there may be something to learn in exploring the approach to teaching of genetics at one school. The Harvard Medical School experience must be seen in the following context:

1. The student body is diverse with regard to prior experience in genetics and career plans. Some of the students have worked in genetics laboratories, whereas others are nonscience majors and have had little prior exposure to genetics. Many are planning academic careers.

2. There is a very large faculty, including a basic science faculty and faculty members associated with several large hospitals in Boston. The basic science faculty includes a department of genetics.

3. There is a separation of 2 preclinical years and 2 clinical years. A problem-based method is used for the preclinical teaching, including lecture and case-based materials, as well as limited clinical exposure. The clinical teaching in years 3 and 4 takes place at multiple hospitals.

4. Courses in the first 2 years occur in blocks of 3 to 12 weeks, during which the students are devoted essentially full-time to a given course. Each of the courses is designed to be integrated with several related disciplines. Genetics is taught as a 51/2-week block integrated with developmental and reproductive biology.

\section{Genetics, developmental and reproductive biology (GDRB)}

This course is taught during the spring of the first year and is attended by all Harvard Medical School students, as well as first-year Harvard Dental School students. It occurs over a 51/2week period, during which time classes meet 5 days a week. There are tutorial sessions Mondays, Wednesdays, and Fridays from 8:30 to 10:00 AM, followed by lectures from 10:15 AM to 12:30 PM. On Tuesdays and Thursdays, there are lectures from 8:30 AM to $12: 30 \mathrm{PM}$.

Approximately $40 \%$ of the lectures are devoted to genetics, $40 \%$ to developmental biology, and 10\% to reproductive biology. Many of the developmental and reproductive biology lectures, however, address topics in genetics, such as disorders of sexual differentiation and genetic control of early development. The lecture schedule is designed to integrate the three topics. For example, the first week includes gametogenesis and early development, with discussion of the basic features of gene structure and function, as well as chromosomes and chromosomal abnormalities.

Although the genetics lectures are designed to introduce basic concepts, each is placed in a clinical context. In part, this is done by liberal use of clinical examples, but it is also done in part by introducing the students to patients and families who are dealing with a genetic disorder. These "clinics" include oculocutaneous albinism (to introduce the concept of the inborn error of metabolism), Down and Turner syndromes (chromosomal anomalies), Smith-Lemli-0pitz syndrome (a developmental disorder due to a block in cholesterol metabolism), Duchenne muscular dystrophy (molecular basis of a genetic disorder and molecular diagnosis), spina bifida (multifactorial inheritance), thalassemia and sickle cell anemia (population genetics), and neurofibromatosis (cancer genetics). The guests who are invited to participate are people who are willing to tell their stories before a large group and, for the most part, have been doing this regularly, often for more than a decade. Their motivation is to communicate their experiences to students at an early stage of their medical education, both to teach them about their disorder and to sensitize the students to both favorable and unfavorable experiences they have had with the medical profession. Students volunteer to interview these "patients" and meet with them for the hour before class begins. In the class, the course director first gives a 10- to 15-minute overview of the condition so that students have a basic familiarity with the disorder. The student and the guest then come to the front of the room and a 15- to 20minute interview ensues. The student is instructed not to conduct a standard medical interview, and no effort is made to point out physical features or do an examination. Rather, the discussion focuses on how the diagnosis was made, what kind of counseling was provided, and how the disorder has impacted the guest's day-to-day life. Following the interview, the class is invited to ask questions. These sessions have proved to be the most popular part of the course. They focus on the human impact of genetic disease and provide the students with 
what may be their first contact with patients as real people. The sessions also serve to whet student appetite to understand the underlying genetic mechanisms.

Tutorials consist of groups of eight students with a faculty tutor. The tutors are all faculty members with academic appointments of instructor and above; postdoctoral fellows, nurses, and counselors do not serve as tutors. Tutorials meet Monday, Wednesday, and Friday each week of the course from 8:30 to 10:00 AM. The tutors meet once a week as a group to go over the next week's case and to discuss progress in the course in general. These meetings provide a major impetus for faculty to serve as tutors, because they provide a forum for faculty members with a common interest in genetics to get to know one another.

A single case serves as the basis for discussion for an entire week. Students are provided the first page in the course syllabus and are expected to read and think about the problem before the Monday tutorial. Additional pages of the case are handed out by the tutor page by page, so that the case unfolds gradually. Cases are intended to introduce new material and do not repeat material covered in lectures. They are designed to stimulate the students to focus on a clinical problem and to explore underlying biological mechanisms as well as approaches to medical decision-making and ethical issues. There is a limited set of reference materials available in the tutorial rooms. Students keep track of questions that arise during a session, and volunteers research answers between sessions. The primary role of the tutor is to be sure that the discussion remains on track but not to answer questions or lecture. Ideally, the tutor remains a relatively quiet member of the group, letting students run the session as much as possible. Tutorial cases during the past year focused on velocardiofacial syndrome (chromosome 22 deletion), an XY phenotypic female, HoltOram syndrome, hemochromatosis, and familial breast cancer.

Student evaluation includes a midterm problem set and final examination, as well as written evaluations by the tutors of tutorial performance. The midterm problem set is a take-home examination, over 1 week, which is graded by the tutors. The major goal is to identify students who are having difficulty with the course material and require additional help. The final examination is also a take-home, over 24 hours, and is graded by hired graders. Both exams are in a short answer format; multiple choice questions are not used. For the past 2 years, there have been questions that require the students to log on to Internet sites such as GeneTests, GeneClinics, and OMIM. This has introduced students to these important computer resources.

\section{Integration of genetics into clinical clerkships}

The GDRB course has a significant visibility in the first-year curriculum, but after that, students have no further formal contact with medical genetics. Only a very small minority choose to take a genetics elective in the third or fourth year. Over the past year, however, we have been experimenting with a way to incorporate genetics into the third-year clerkship in medicine at Brigham and Women's Hospital in Boston.

This clerkship extends over 3 months, with approximately 14 students involved for each 3-month block. This is only one of several sites where Harvard Medical School students may do their medicine clerkship. During the first month, all 14 students work on the inpatient service. During the second month, half do outpatient clinics while half stay on the inpatient service, and during the third month, the two groups switch. Therefore, all students do 2 inpatient months and 1 outpatient month during the rotation.

Since July 2000, we have conducted an exercise to include genetics in the curriculum of this clerkship. A handout has been provided, which is incorporated into the written material provided to the students on the first day. This provides a description of the exercise, a primer on family history-taking and inheritance, a description of genetic testing resources, and Internet links. The students meet with a medical geneticist, who reviews with them the overall approach to genetics in medical practice. For the first month, students are asked to use a template to take a family history for all patients whom they admit to the hospital. These are handed in to the medical geneticist near the end of the month, and the students meet with the geneticist to review these pedigrees. Selected pedigrees are discussed with the group, with the student who handed in a particular pedigree leading the discussion of that family. The students are asked to comment on the material handed in, answering these three questions:

1. Identify one or more traits segregating in the family, and discuss possible mode(s) of genetic transmission.

2. Would recognition of the patient's risk at an earlier time have enabled interventions that might have avoided the need for this hospitalization?

3. Are there issues that should be discussed with other family members regarding their own risks? How would you explain the significance of these issues to their own health?

The major goal of this exercise is to illustrate how genetics can contribute to routine medical practice, and also to help the students recognize the challenges faced in collecting family history information, particularly in the setting of acute hospitalization. The exercise has also provided an interesting "snapshot" of the genetics content that accompanies inpatient hospitalization. Some single gene disorders have appeared (cardiomyopathy, thrombophilia, myotonic dystrophy), as well as some multifactorial disorders with a strong hereditary component (breast or colon cancer, for example).

Students who are doing their outpatient months are presented with a different exercise. Each is assigned to attend one clinic that has a focus on genetics once a week for 4 weeks. The clinics are a general genetics clinic, a colon cancer genetics clinic, a breast cancer genetics clinic, a nephrology clinic with a focus on hereditary renal disorders, and two hematology clinics (one that focuses on coagulopathies and one that focuses on globin disorders). The same student will attend one of these 
clinics four times. At the end of the month, the students are asked to hand in a one-case vignette and to address a set of questions. They also meet with the medical geneticist, and students describe their experience in the clinic to their colleagues and present their cases to one another. The questions are as follows:

1. What is the range of genetic disorders covered in this clinic?

2. What kinds of personnel were involved in providing patient care in the clinic?

3. Was genetic information incorporated into the medical records or kept in a separate file?

4. What was the role of genetic testing in this clinic, and how were data reported to patients?

These two exercises have emphasized the importance of engaging third-year students in genetic issues in real patients. Part of the time they focus on the genetics content of hospitalized general medicine patients, and part of the time they see patients in a clinic with an emphasis on genetics. It will be important to measure the impact of this program on knowledge, skills, and attitudes, which is a plan for the coming year.

Genetics has captured the attention of the public, and there are high expectations for the incorporation of new knowledge of the human genome into medical practice. This will require an increased understanding of the principles of genetics and acquisition of specific skills on the part of all physicians. The transition of genetics from the study of the obscure to the commonplace is taking place very rapidly-in some cases, faster than the system of medical education can adapt. It will be important in the coming years to test various models of genetics education in the medical school curriculum and to measure outcomes that document efficacy. The practice of medicine will be gradually reshaped, and the medical school curriculum will need to use creative new measures to keep up with this rapid pace of change.

\section{References}

1. Collins FS. Shattuck lecture: medical and societal consequences of the Human Genome Project. N Engl J Med 1999;341:28-37.

2. Holtzman NA, Marteau TM. Will genetics revolutionize medicine? N Engl J Med 2000;343:141-144.

3. Kaback M, Lim-Steele J, Dabholkar D, Brown D, Levy N, Zeiger K. Tay-Sachs disease: carrier screening, prenatal diagnosis, and the molecular era. JAMA 1993;270:23072315 .

4. Rowley PT, Loader S, Sutera CJ, Walden M, Kozyra A. Prenatal screening for hemoglobinopathies, III: applicability of the health belief model. Am J Hum Genet 1991;48: 452-459.

5. Rowley PT, Loader S, Sutera CJ, Walden M, Kozyra A. Prenatal screening for hemoglobinopathies, I: a prospective regional trial. Am J Hum Genet 1991;48:439-446. 